\title{
Factores de riesgo de enfermedades crónicas no transmisibles en estudiantes que ingresan a la carrera de enfermería en Ciudad Victoria, Tamaulipas
}

\author{
Flor Esthela Carbajal-Mata*, Patricia Rivera-Vázquez, Olga Lidia Banda-González \\ y Gabriela Maldonado-Guzmán
}

Facultad de Enfermería Victoria, Universidad Autónoma de Tamaulipas, Ciudad Victoria, Tamaulipas, México

\begin{abstract}
Objective: To evaluate the presence of risk factors for chronic non-communicable diseases among students enrolled in the Nursing School of Victoria, Tamaulipas, for the period of January-May, 2016. Methods: Descriptive, transversal study including 139 newly enrolled students from a Nursing School. Students were evaluated at the beginning of the school period. Health data were obtained from the students' individual clinical files. Results: Blood pressure and body mass index values were within normal ranges. Most students did not perform a physical activity; $24.5 \%$ had a family history of diabetes mellitus and $13.7 \%$ of arterial hypertension. Conclusions: Although results indicate that the new student population have a good health condition, it is necessary to reinforce good lifestyles, such as physical activity and good nutrition, to prevent the appearance of chronic non-communicable diseases. (Hosp Med Clin Manag. 2019;12:64-8)

Corresponding author: Flor Esthela Carbajal-Mata, fcarbajal@docentes.uat.mx
\end{abstract}

Key words: Students. Chronic Disease. Risk factors.

\section{RESUMEN}

Objetivo: Evaluar la presencia de los factores de riesgo de enfermedades crónicas no transmisibles en los estudiantes que ingresan a la Facultad de Enfermería Victoria en el periodo enero-mayo 2016. Métodos: Estudio descriptivo, transversal. Participaron 139 estudiantes de nuevo ingreso a la Facultad de Enfermería Victoria, valorados al inicio del periodo escolar, cuya información fue reportada en el expediente clínico institucional. Resultados: Los valores de presión arterial y el índice de masa corporal se encontraron dentro de los rangos normales. Se identificó que la mayor parte de los estudiantes no realizan actividad física. El 24.5\% tienen antecedentes familiares de diabetes

\section{Correspondencia:}

${ }^{*}$ Flor Esthela Carbajal-Mata

E-mail: fcarbajal@ docentes.uat.mx
Date of reception: $09-08-2018$

Date of acceptance: 11-06-2019

DOI: 10.24875/HMCM.19000146 
mellitus y el $13.7 \%$ de hipertensión arterial. Conclusiones: Si bien los resultados indican que en general la población estudiantil de nuevo ingreso muestra un cuadro saludable, es necesario reforzar los buenos estilos de vida, como la actividad física y la buena alimentación, con la finalidad de prevenir el incremento de la prevalencia de enfermedades crónicas no transmisibles.

Palabras clave: Estudiantes. Enfermedades crónicas. Factores de riesgo.

\section{INTRODUCCIÓN}

La vida universitaria no solo contempla el cumplimiento de las exigencias académicas, sino que también existe una gran cantidad de cambios de adaptación relacionados con la alimentación, la actividad física, el descanso, el entretenimiento y el estrés, entre otros, lo que se refleja en el estado emocional y de salud del estudiante1. La alteración de estos elementos implica el desarrollo de problemas crónicos de salud a temprana edad tales como la diabetes mellitus (DM), la obesidad, la hipertensión arterial (HTA) y el desarrollo de hábitos adictivos como el consumo de alcohol, tabaco y drogas ilícitas².

Las enfermedades crónicas no transmisibles (ECNT) son padecimientos de larga duración y de evolución progresiva, que suele ser lenta y dolorosa, siendo principalmente las enfermedades cardiovasculares, el cáncer, las enfermedades respiratorias crónicas y la DM las causas de defunción más importantes del mundo, con un $63 \%$ de muertes anuales, en donde aproximadamente el $80 \%$ de estas suceden en países de ingresos bajos y medios $^{3}$. Se supone que los jóvenes gozan de buena salud; sin embargo, las estadísticas por mortalidad prematura, morbilidad y lesiones en este grupo de población son considerables, debido a que afectan la capacidad de crecer y desarrollarse en forma óptima. El consumo de tabaco y alcohol, aunado a la disminución de actividad física, el inicio de actividad sexual sin protección, y violencia expuesta, los colocan en una situación de riesgo con implicaciones en la edad adulta. Por ello es necesario que durante esta etapa se fomente la adopción de medidas contra los riesgos sanitarios que prevengan los problemas de salud en la edad adulta y, por ende, a las generaciones futuras ${ }^{4}$.

En 2015, la información de la Encuesta Intercensal mostró que la población joven de 15 a 29 años en México aumentó hasta los 30.6 millones, lo que representan poco más de la cuarta parte $(25.7 \%)$ de la población nacional ${ }^{5}$. Del total de la población joven, el $35.1 \%$ son adolescentes (15 a 19 años), el 34.8\% son jóvenes de 20 a 24 años y el $30.1 \%$ tienen de 25 a 29 años de edad. Tamaulipas es una de las entidades con más bajo porcentaje de jóvenes, en el 2015 se reportó que el $24.4 \%$ de la población tiene entre 15 y 29 años de edad 6 .

En lo que respecta al consumo de alcohol y tabaco como factores predisponentes, en México, la Encuesta Nacional de Adicciones (2011) señala que el consumo de alcohol alguna vez en la vida en población de 18 a 34 años fue del $42.9 \%$, mientras que en el último año fue del $30 \%$ y en el último mes, del $14.5 \%$. El $14.5 \%$ de la población presenta un consumo alto de alcohol: el $0.2 \%$ refirió un consumo diario de alcohol, el $1 \%$ son consumidores consuetudinarios y el $4.1 \%$ se refiere a adolescentes y jóvenes con dependencia del alcohol ${ }^{7}$. Por otro lado, los antecedentes heredofamiliares influyen de manera diferente en la salud de las personas y pueden aumentar el riesgo de padecer ciertas enfermedades a temprana edad, como la HTA y la DM¹. Entre los factores de riesgo para desarrollar ECNT más comunes encontrados en población universitaria están los antecedentes hereditarios, la inactividad física, el sobrepeso, la obesidad y el sedentarismo ${ }^{8-11}$. Aunado a estos factores, también se han destacado en universitarios la presencia de hábitos alcohólicos y tabáquicos, tanto en hombres como en mujeres ${ }^{12-14}$.

El Institute of Health Metrics de la Universidad de Washington ${ }^{19}$ indica que los principales factores de riesgo a la salud que generan años de vida saludables perdidos (AVISA) en personas de 15 a 49 años en Tamaulipas, al 2016, son un índice de masa corporal (IMC) elevado (1,722.26 AVISA/100,000 hab), seguido de la glucosa elevada (1,636.92 AVISA/100,000 hab), el consumo de alcohol (1,452.92 AVISA/100,000 hab), la insuficiencia renal (630.47 AVISA/100,000 hab) y la presión sistólica elevada (617.42 AVISA/100,000 hab). El consumo de drogas ocupa la sexta posición (600.57 AVISA/100,000 hab), mientras que el consumo de tabaco la 12. ${ }^{a}$ (315.62 AVISA/100,000 hab) ${ }^{19}$. 
En estudiantes universitarios aparentemente sanos se considera de interés investigar los factores de riesgo presentes, ya que en la actualidad hay profundos cambios en el estilo de vida y de alimentación en la población joven. Esta situación se ve reflejada en el aumento del consumo de comida rápida, la falta de actividad física y el incremento del consumo del alcohol y tabaco, por lo que los factores de riesgo no deben ser considerados simplemente como aditivos, sino como multiplicadores ${ }^{15,16}$. Consecuentemente, un gran número de factores de bajo riesgo pueden ser tan importantes como uno de alto riesgo; esto permite adelantar que ciertos factores son potencialmente modificables. Por ello, la presente investigación tiene como objetivo evaluar la presencia de los factores de riesgo de ECNT en los estudiantes de nuevo ingreso a la Facultad de Enfermería Victoria del periodo enero-mayo 2016.

\section{MÉTODOS}

Investigación descriptiva, no experimental y transversal ${ }^{17}$, que buscó establecer la presencia o no de factores de riesgo para padecer ECNT en estudiantes universitarios. La población estuvo conformada por 139 estudiantes que ingresaron a primer semestre a la Facultad de Enfermería Victoria en el periodo eneromayo 2016, quienes fueron valorados al inicio del periodo escolar. El $73 \%(n=101)$ fueron del sexo femenino, con edades comprendidas entre los 17 y 26 años.

La recolección de la información se realizó mediante el uso de un expediente clínico oficial de la Unidad de Atención Integral de Enfermería, del cual se empleó la información correspondiente a la edad, peso, talla, el diagnóstico nutricional antropométrico, antecedentes familiares, actividad física y consumo de tabaco y alcohol. Para el diagnóstico nutricional antropométrico se empleó el IMC, siguiendo los criterios de valoración según la NOM-043-SSA2-2005, considerando como sobrepeso cuando el IMC del estudiante fuera entre $25 \mathrm{y}$ $29.99 \mathrm{~kg} / \mathrm{m}^{2}$ y obesidad cuando el IMC fuera mayor a $30 \mathrm{~kg} / \mathrm{m}^{2}$. Se utilizaron los criterios de la NOM-030SSA2-2009 para la evaluación de la presión arterial (PA), clasificando como hipertensos a aquellos cuya PA sistólica fuera $\geq 140 \mathrm{mmHg} O$ una PA diastólica $\geq 90 \mathrm{mmHg}$.

Para determinar si el estudiante era sedentario o no, se consideró si respondían «ningún tipo de actividad física»
Tabla 1. Promedio de las variables continuas distribuidas según el sexo

\begin{tabular}{lcc}
\hline Variable & \multicolumn{2}{c}{ Sexo } \\
\cline { 2 - 3 } & Masculino & Femenino \\
\hline Distribución, $\mathrm{n}(\%)$ & $38(27.34)$ & $101(72.66)$ \\
Edad, años & 18.26 & 18.46 \\
PA sistólica, mmHg & 110.78 & 109.90 \\
PA diastólica, mmHg & 75.52 & 73.16 \\
Peso, kg & 64.60 & 65.40 \\
Talla, $\mathrm{m}$ & 1.62 & 1.60 \\
IMC, $\mathrm{kg} / \mathrm{m}^{2}$ & 24.41 & 25.16 \\
\hline
\end{tabular}

PA: presión arterial; IMC: índice de masa corporal.

en el punto correspondiente del cuestionario (¿Qué actividad realiza durante su tiempo libre?). Se consideraron como fumadores aquellos estudiantes que en ese momento fumaban o hubiesen fumado en los últimos dos años, al menos un cigarrillo por día y durante un año ininterrumpido. Se consideró un consumo positivo aquellos que referían ingerir alcohol con una frecuencia diaria o semanal en los últimos tres años, por lo menos durante un año ininterrumpido. Los datos fueron analizados descriptivamente, empleando el paquete estadístico SPSS ${ }^{\circ}$ V. 20.

El estudio contó con el dictamen favorable del Comité de Ética e Investigación de la Facultad de Enfermería Victoria, de la Universidad Autónoma de Tamaulipas (UAT). Posteriormente se solicitó autorización a la Unidad de Atención Integral de Enfermería de la misma Facultad, para evaluar al grupo de estudiantes previa firma de consentimiento informado. En dicho lugar se procedió a la entrevista y valoración del grupo de estudio.

Este estudio fue sustentado en la Ley General de Salud de México, Reglamento de la Ley General de Salud en Materia de Investigación para la Salud.

\section{RESULTADOS}

La tabla 1 muestra las características generales de los estudiantes valorados, el valor promedio para las variables de edad, PA sistólica, PA diastólica, peso, talla e IMC. Tanto la PA sistólica como la diastólica y el IMC muestran valores promedio, que indicarían que se encuentran dentro de un cuadro de «no hipertenso" y un estado nutricional aceptable. 
Tabla 2. Hábitos tabáquicos, alcohólicos y actividad física

\begin{tabular}{|c|c|c|c|}
\hline \multirow[t]{2}{*}{ Variable } & \multicolumn{2}{|c|}{ Sexo } & \multirow{2}{*}{$\begin{array}{c}\text { General } \\
\text { n ( } \%)\end{array}$} \\
\hline & $\begin{array}{c}\text { Masculino } \\
\text { n (\%) }\end{array}$ & $\begin{array}{c}\text { Femenino } \\
\mathrm{n}(\%)\end{array}$ & \\
\hline \multicolumn{4}{|l|}{ Consumo de tabaco } \\
\hline - Consumen & $7(18.40)$ & $7(6.90)$ & $14(10.10)$ \\
\hline - No consumen & $31(81.60)$ & $94(93.10)$ & $125(89.90)$ \\
\hline \multicolumn{4}{|l|}{ Consumo de alcohol } \\
\hline - Consumen & $6(15.70)$ & $4(3.90)$ & $10(7.10)$ \\
\hline - No consumen & $32(84.30)$ & $97(92.90)$ & $129(92.90)$ \\
\hline \multicolumn{4}{|l|}{ Actividad física } \\
\hline - Realizan & $19(50.00)$ & $14(13.80)$ & $33(23.70)$ \\
\hline - No realizan & $19(50.00)$ & $87(86.20)$ & $106(76.30)$ \\
\hline
\end{tabular}

En cuanto a la presencia de hábitos tabáquicos y/o alcohólicos (Tabla 2), se encontró que el 10.1\% del total de los estudiantes fumaban ocasionalmente de 1 a 2 cigarrillos; en cuanto al consumo de bebidas alcohólicas ocasionales, este se presentó mayormente en los varones, con un 15.7\%. La actividad física mostró un elevado porcentaje de sedentarismo, con un $76.3 \%$ del total de los estudiantes, siendo más elevado en el sexo femenino, con un $86.2 \%$.

En relación a la presencia de antecedentes de ECNT en familiares directos (padre, madre, abuelos) de la población de estudiantes, se encontró un porcentaje importante de DM, en el 24.5\%, seguido de HTA y cáncer (Tabla 3).

\section{DISCUSIÓN}

El presente estudio tuvo como objetivo evaluar los factores de riesgo de ECNT en un grupo de estudiantes de nuevo ingreso a la carrera de Enfermería y lograr identificar los posibles detonantes que pudieran deteriorar su salud a lo largo de su vida académica, ya que son personas jóvenes que en un futuro cercano serán nuestros cuidadores y deben predicar con el ejemplo. Ellos se encuentran en un espacio crítico de su vida debido a que la presión universitaria tiende a disminuir la actividad física y fácilmente se aumenta el peso debido a las nuevas prácticas alimentarias que adquieren, lo que resulta en sobrepeso y obesidad. Así mismo, el contexto que les rodea puede incluso iniciar, si no es que ya han iniciado, hábitos tabáquicos y alcohólicos, entre otros².

El grupo de universitarios estudiado muestra que las variables analizadas se encuentran en rangos aparentemente normales (peso, talla, IMC y PA). Sin embargo, al analizar
Tabla 3. Antecedentes de enfermedades crónicas no transmisibles en familiares directos

\begin{tabular}{lc}
\hline $\begin{array}{l}\text { Enfermedad presentada en la } \\
\text { familia del estudiante }\end{array}$ & $\begin{array}{c}\text { General } \\
\mathbf{n}(\%)\end{array}$ \\
\hline Cáncer & $5(3.50)$ \\
Diabetes mellitus & $34(24.50)$ \\
Hipertensión arterial & $19(13.70)$ \\
Ninguna & $81(58.30)$ \\
\hline
\end{tabular}

la presencia de hábitos tabáquicos y/o alcohólicos se encontró el hábito positivo observado más en los varones, tal como lo muestran algunos estudios ${ }^{12-14}$. En ellos se observó que tanto los hombres como las mujeres ya iniciaron en este hábito, lo que también pudiera representar el nuevo contexto en el que se desenvolverán los próximos 4 a 5 años de carrera universitaria, y en donde significativamente se observa más en los varones. En cuanto a la actividad física, se observó un alto porcentaje de sedentarismo, más elevado en el sexo femenino; este es uno de los factores de riesgo más comunes para desarrollar ECNT, ya que esta inactividad física propicia la presencia de sobrepeso y obesidad, como lo demostraron algunos estudios $^{8,9,11}$. Esta inactividad física generalmente es debida a la carga horaria de los universitarios, así como los cambios drásticos en la alimentación saludable y, por ende, la falta de tiempo para preparar los alimentos en casa, ya que es más fácil realizar su adquisición en los locales de calle, aunado al hecho de que un alto porcentaje de los estudiantes de esta Facultad de Enfermería son foráneos.

Sin duda uno de los detonantes para desarrollar estas enfermedades crónicas se encuentra en los malos hábitos alimenticios ${ }^{11}$, sobre todo debido al cambio drástico que sufre el estudiante que recién ingresa al grado 
universitario y que propician el sobrepeso y la obesidad. Aunado a los factores antes mencionados, en adultos de 20 años o más de edad se ha observado que la prevalencia del aumento de peso es mayor en las mujeres ${ }^{18}$.

Así mismo, se destacan los antecedentes heredofamiliares, en primer lugar, la DM, seguida de la HTA y en tercer lugar la presencia de cánceres en familiares directos, como lo demuestran Oviedo, et al. ${ }^{10}$, en donde la HTA y la obesidad predominan en los padres de los estudiantes. $Y$ estos antecedentes definitivamente son un detonador a futuro para la presencia de las ECNT en los nuevos egresados del área de la salud, específicamente enfermeros y enfermeras.

Los conocimientos sobre daño a la salud por enfermedades crónicas son importantes y apegados a la evidencia científica. Los hallazgos resaltan la necesidad de mantener y reforzar los proyectos intervencionistas que ya se llevan a cabo, para incidir en la prevalencia de estas enfermedades crónicas entre los jóvenes universitarios de reciente ingreso.

\section{CONCLUSIONES}

Finalmente, se concluye que esta población de universitarios que ingresan a la Facultad de Enfermería Victoria aún se encuentra aparentemente sana, en lo que respecta a valoración de peso/talla y PA. Sin embargo, se identificó la presencia de factores de riesgo para ECNT, y que tienen su mayor detonante en el sedentarismo y el antecedente familiar. En menor grado quedó el consumo de bebidas alcohólicas y el hábito tabáquico, en donde se detectó que un pequeño porcentaje de los nuevos estudiantes ya tenía estas dos adicciones a su ingreso. A este respecto, debe prevenirse que el resto de los estudiantes no las adquiera durante su paso por la universidad o, también importante, en su defecto disminuyan su consumo. En virtud de estos hallazgos tempranamente encontrados, el personal del área de la salud de la Facultad de Enfermería Victoria implementa programas de promoción de estilos de vida saludables, a fin de prevenir y detectar precozmente factores de riesgo que pudieran influir en la aparición de las ECNT.

\section{BIBLIOGRAFÍA}

1. Ibáñez E, Thomas Y, Bicenty A, Barrera J, Martínez J, Gerena R. Cambios de hábitos alimentarios de los estudiantes de odontología de la Fundación Universitaria San Martín de Bogotá, Colombia. NOVA. 2008;6(9):27-34.

2. Sánchez ML, Álvarez A, Flores T, Arias J, Saucedo M. El reto del estudiante universitario ante su adaptación y autocuidado como estrategia para disminuir problemas crónicos degenerativos. Educ. Salud Bol. Cient. Inst. Cienc. Salud Univ. Autónoma Estado Hidalgo. 2014;2(4).

3. 10 datos sobre las enfermedades no transmisibles [Internet]. Organización Mundial de la Salud; 2013. Disponible en: https://www.who.int/features/ factfiles/noncommunicable_diseases/es/

4. Adolescentes: riesgos para la salud y soluciones [Internet]. Organización Mundial de la Salud; 2017. Disponible en: http://www.who.int/mediacentre/ factsheets/fs345/es/

5. INEGI: Estadísticas a propósito del día internacional de la juventud (15 a 29 años) [Internet]. México: Instituto Nacional de Estadística y Geografía 2019. Disponible en: http://consulta.mx/index.php/estudios-e-investigaciones/otros-estudios/item/702-inegi-estadisticas-a-proposito-del-dia-internacional-de-la-juventud-12-de-agosto

6. La OPS/OMS y la Sociedad Latinoamericana de Nefrología llaman a prevenir la enfermedad renal y a mejorar el acceso a tratamiento [Internet]. Organización Panamericana de la Salud; 2015. Disponible en: http://www.paho. $\mathrm{org} / \mathrm{hq} /$ index php?option=com content\&view=article\&id=10542\%3A2015opsoms-sociedadlatinoamericana-nefrologia-enfermedad-renal-mejorartratamiento\&ltemid=1926\&lang=es Recuperado en mayo de 2016.

7. Encuesta Nacional de Adicciones 2011: Reporte de alcohol [Internet]. México: Instituto Nacional de Psiquiatría Ramón de la Fuente Muñiz; 2012. Disponible en: http://www.conadic.salud.gob.mx/pdfs/ENA_2011_ALCOHOL.pdf

8. Arenas G, Torres MA, Santillán M, Robles AL. Factores de riesgo para enfermedades crónicas en universitarias del área de la salud. Rev Enfermería Universitaria ENEO-UNAM. 2008;5(2):16-21.

9. McColl P, Macarena A, Aros J, Lastra A, Pizarro C. Prevalencia de factores de riesgo de enfermedades crónicas no transmisibles en estudiantes de medicina de la Universidad de Valparaíso. Rev Chilena Pediatr. 2002;73(5):478-82.

10. Oviedo G, Morón de Salim A, Santos I, Sequera S, Soufrontt G, Suárez P, et al. Factores de riesgo de enfermedades crónicas no transmisibles en estudiantes de la carrera de Medicina: Universidad de Carabobo, Venezuela. Nutr Hosp. 2008;23(3):288-93.

11. Pulido C, Reyes N, León A, Cárdenas J, Rivera S, Rodríguez AY. Factores de riesgo de enfermedades crónicas no transmisibles en estudiantes de ciencias básicas de la escuela de medicina de la Universidad Pedagógica y Tecnológica de Colombia [Internet]. Rev.salud.hist.sanid.on-line 2015; 0(1) (enero-junio). Disponible en: https://www.researchgate.net/profile/Cristian Pulido-Medina/publication/298820526 FACTORES DE RIESGO DE ENFERMEDADES_CRONICAS_NO_TRANSMISIBLES_EN_ESTUDIANTES DE_CIENCIAS_BASICAS_DE_LA_ESCUELA_MEDICINA_DE_LA_UNIVERSIDAD PEDAGOGICA/links/56ecd52108aea35d5b98607d.pdf

12. Telumbre-Terrero JY, Esparza-Almanza SE, Alonso-Castillo BA, AlonsoCastillo MTJ. Consumo de alcohol y tabaco en estudiantes de enfermería [Internet]. Revenf. 2016;30:1-16. Disponible en: https://www.scielo.sa.cr/ pdf/enfermeria/n30/1409-4568-enfermeria-30-00001.pdf

13. Mantilla-Toloza SC, Villamizar CE, Peltzer K. Consumo de alcohol, tabaquismo y características sociodemográficas en estudiantes universitarios. Rev Univ Salud. 2016:18(1):7-15.

14. Fabelo JR, Iglesias S, Cabrera R, Maldonado, MT. Consumo de tabaco y alcohol entre los estudiantes de ciencias de la salud en Cuba y México. MEDICC Rev. 2013;15(4).

15. López-Azpiazu I, Sánchez-Villegas A, Johansson L, Petkeviciene J, Pretalla $\mathrm{R}$, Martínez-González M. Disparities in food habits in Europe: systematic review of educational and occupational differences in thein take of fat. $J$ Hum Nutr Diet. 2003:16(5):349-64.

16. Hill J, Wyatt H, Reed G, Peters JC. Obesity and the environment. Where do we go from here? Science. 2003;299:853-5.

17. Polit D, Hungler BP. Investigación científica en ciencias de la salud. México: McGrawHill-Interamericana de México; 2000.

18. Encuesta Nacional de Salud y Nutrición de Medio Camino 2016. Informe final de resultados [Internet]. México: Secretaría de Salud; 2016. Disponible en: http://fmdiabetes.org/wp-content/uploads/2017/04/ENSANUT2016-mc.pdf

19. Health Data Visualization. Datos AVISA por factores de riesgo de población de 15 a 49 años en Tamaulipas 2016 [Internet]. EE.UU.: University of Washington, GBD Compare. Disponible en: https://vizhub.healthdata.org/gbd-compare/\# 